\title{
The H2020 OCRE Project Opens the Gates of the Commercial Cloud and EO Services Usage to the Research Community
}

\author{
José Manuel Delgado Blasco ${ }^{\text {a* }}$, Antonio Romeo ${ }^{a}$, David Heyns ${ }^{\mathrm{b}}$, Joao Fernandes ${ }^{\mathrm{c}}$, \\ Rob Carrillo ${ }^{\mathrm{d}}$, Natassa Antoniou ${ }^{\mathrm{e}}$, Lefteris Mamais ${ }^{\mathrm{f}}$, Marc-Elian Begin ${ }^{\mathrm{g}}$ \\ ${ }^{a}$ RHEA Group, Via di Grotte Portella 6/8, 00044 Frascati, Italy \\ ${ }^{b}$ GÉANT, Amsterdam, The Netherlands \\ ${ }^{c}$ CERN, Geneva, Switzerland \\ ${ }^{d}$ Trust-IT, Pisa, Italy \\ ${ }^{e}$ EARSC, Brussels, Belgium \\ ${ }^{f}$ Evenflow, Brussels, Belgium \\ ${ }^{g}$ SixSq, Geneva, Switzerland
}

\begin{abstract}
Cloud and Earth Observation (EO) based services offer the European Research community a wealth of powerful tools. However, for many researchers these tools are currently out of reach. It is difficult to find and select suitable services. Establishing agreements with cloud and EO service providers and ensuring legal and technical compliance requires specialist skills and takes an inordinate amount of time. Equally, service providers find it difficult to reach and meet the needs of the research community in technical, financial and legal areas. The Open Clouds for Research Environments consortium (OCRE) will change this, by putting in place an easy adoption route. In the autumn of 2019, OCRE will run a pan-European tender and establish framework agreements with service providers who meet the requirements of the research community. 10.000 European research and education institutes will be able to directly consume these offerings via the European Open Science Cloud service catalogue, through ready-to-use agreements. They will not have to run a tender of their own. In addition, to stimulate usage, OCRE will make available 9.5 million euro in service credits (vouchers), through adoption funds from the European Commission. OCRE is a pioneer project without precedence, with potentially high impact in the future EO market activities and evolution of service offering, with the objective to burst the usage of EO commercial services by the research environment.
\end{abstract}

\section{Keywords:}

Commercial Services;

Earth Observation;

Research Community;

Easy Adoption;

Vouchers; Adoption Fund;

EOSC-Hub;

European Commission.

\section{Article History:}

$\begin{array}{llll}\text { Received: } & 18 & \text { December } & 2019 \\ \text { Accepted: } & 10 & \text { March } & 2020 \\ \text { Published: } & 01 & \text { April } & 2020\end{array}$

\section{1- Introduction}

The European Open Science Cloud (EOSC) is a European Commission (EC) initiative started in 2015 [1] that aims at the development an infrastructure providing its users with services using and promoting open science practices, such as the FAIR Data principles [2]. The available infrastructure is being built by aggregating European (EU) services providers.

An EU committee on research endorsed a plan for the cloud's development in May 2018 [3]. The EOSC initiative was officially launched in November 2018, starting to provide access to services via their EOSC Portal [4]. EOSC-hub [5] is a single point for European researchers and innovators to discover, access, use and reuse a wide spectrum of resources for data-driven research from multiple service providers.

\footnotetext{
*CONTACT: J.delgado@rheagroup.com

DOI: http://dx.doi.org/10.28991/esj-2020-01213
}

(C) 2020 by the authors. Licensee ESJ, Italy. This is an open access article under the terms and conditions of the Creative Commons Attribution (CC-BY) license (https://creativecommons.org/licenses/by/4.0/). 
EOSC-hub includes providers from European research infrastructures such as the EGI Federation [6], EUDAT CDI [7], INDIGO-DataCloud [8] among others, to deliver a common catalogue of research data, services and software for research.

The Open Cloud for Research Environment (OCRE) is a Horizon2020 project funded by the EC [9]. The OCRE consortium consists of GÉANT (consortium coordinator), CERN, RHEA and Trust-IT with the contribution of SixSq, as technology provider, together with EARSC and Evenflow, as support to outreach activities, and receives funding from the European Commission, as part of the European Open Science Cloud (see Figure 1).

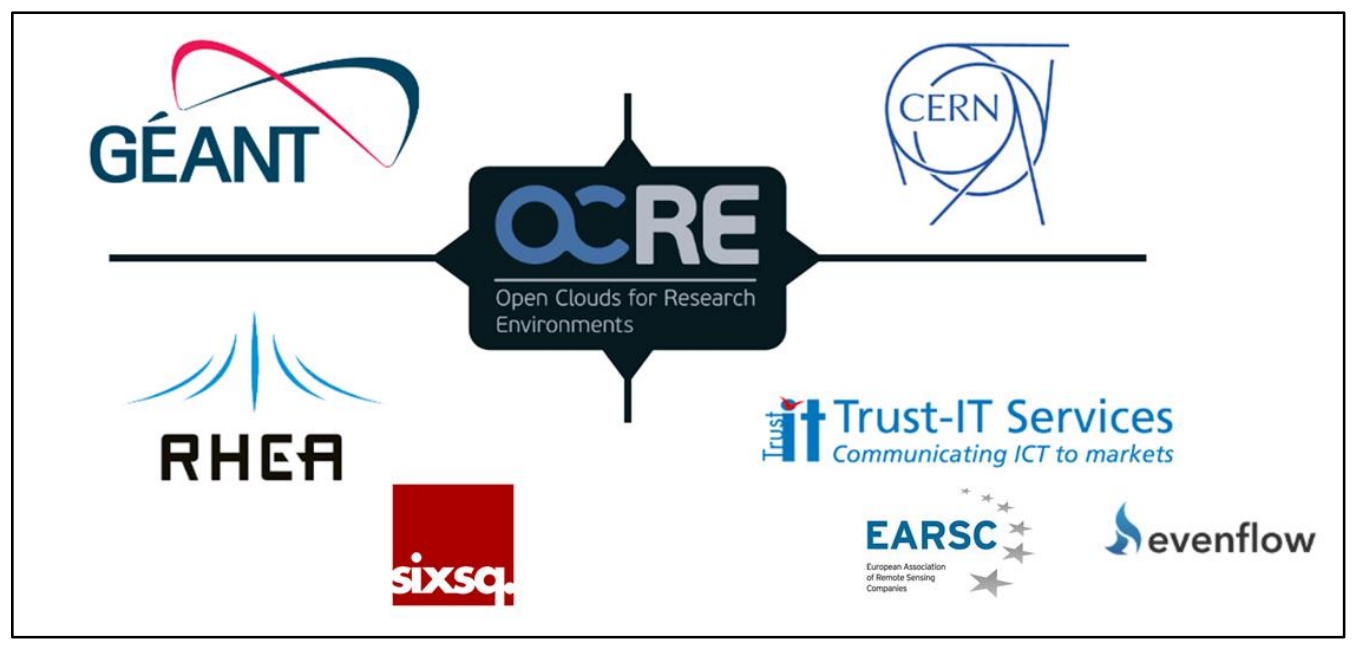

Figure 1. OCRE project and the partners in the consortium.

OCRE is part of EOSC and collaborates with EOSC-hub, where the latter, at the same time, also works closely with eInfraCentral [10], EOSCpilot [11], GÉANT 4.2 [12], OpenAIRE-Advance [13] and the RDA Europe 4.0 projects to deliver a consistent service offer for research communities across Europe. Another examples of projects in synergy on these subjects are EOSC-SYNERGY [14], DEEP Hybrid DataCloud [15], Cos4Cloud [16], EPOSORFEUS [17], DODAS [18] and ENES [19] among others.

This paper introduces the benefits of the OCRE project for providers and for researchers. It will also address how OCRE aggregates community demand and requirements and applies these into a pan-European call-for-competition, for commercial service providers to respond to. These tenders which will be launched in Q3/Q4 of 2019, and it will result in procurement-compliant framework agreements with suitable suppliers.

Moreover, the paper will introduce how researchers, affiliated/not affiliated to institutions will be able to consume the services through ready-to-use contracts, without the need to run their own tender and how OCRE will stimulate usage through an adoption fund.

OCRE is part of the European Open Science Cloud and receives funding from the EC under grant agreement no. 824079.

\section{2- Project Scope}

The EC wants to stimulate the usage of commercial cloud (Type A) and Earth Observation (Type B) services by the European research community, due to their current low usage. Some of the reasons for this low usage of commercial services could be due to the difficulty that faces the research community to find proper services and, also the difficulty to reach legal and technical agreements between companies and research institutions. This latter normally takes enormous amount of time and not always reaches final agreements, hence discouraging both researchers and companies for future activities.

This is exactly the scope of the OCRE project, to create agreements directly with the service providers and provide a list of suitable services, searchable online in the EOSC market place, lowering all the barriers that until now restricted the usage of commercial services by the research community.

Additionally and for stimulating the usage of services which will be compliant with standards and requirements requested in the tender, the European Commission puts available over 9 million euros to distribute among the researchers and institutions to use services free-at-the-point-of-the-user.

The project started in Q1 2019, and will end in $31^{\text {st }}$ December 2019 and. During 2019, first selected researchers will be able to benefit from cloud commercial services of an amount of 500,000 to start raising the awareness of the project using contracts that GÉANT has already in place from previous projects. Just after the tenders will be closed 
and the contracts signed in Q1 2020, the EU research community will start consuming the remaining budget on the cloud and EO services that will be on-boarded by the OCRE project.

\section{2-1-The Role of the NRENS}

In this project, it is important that as many researchers and institutions as possible are able to benefit from the advantages offered by the OCRE project and hence, the National Research and Education Network from each country (national NRENs) have an important role. They represent a door that can be opened for many institutions, which are not aware of the OCRE project belonging to a NREN, if their NRENs register in OCRE. By registering, their members can access and request to benefit from OCRE in different ways depending of the level of participation of the different NRENs.

Moreover, GÉANT is in direct contact with the different NRENs, as they are collaborating since many years in other areas, such network provisioning, but here, in OCRE, they will collaborate to enable many institutions to use the cloud and EO services that will be on-boarded in OCRE. After the closing deadline for NREN registration, 39 NRENs got registered in OCRE (see Figure 2 their geographical distribution).

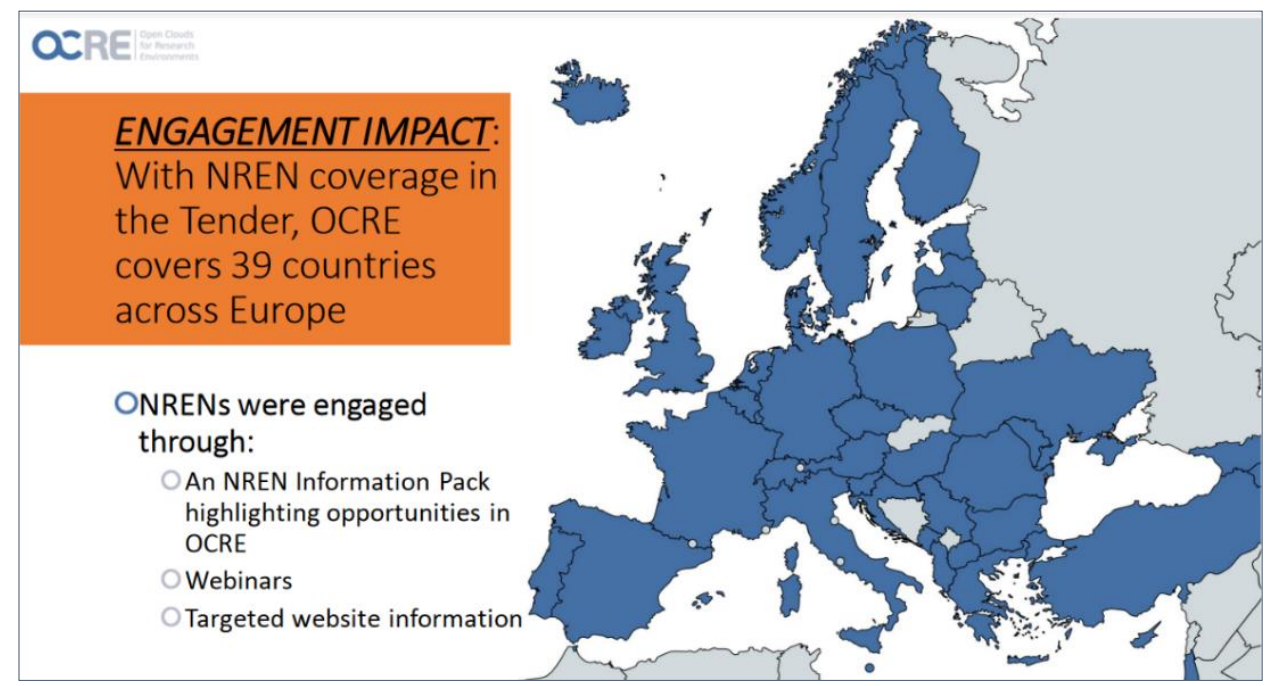

Figure 2. Geographical distribution of registered NREN in OCRE.

\section{2-2-Users}

The project foresees three main types of users:

- Individual researchers:

○ Funding mechanism with $100 \%$ EC funds;

$\circ$ Researchers with EU affiliation;

○ Wave 1: vouchers;

○ Scientific organisations for the voucher screening and distribution.

- Individual institutions:

○ Funding mechanism with $100 \%$ EC funds;

- EU research and education institutions;

$\circ$ Wave 2: vouchers;

o Screening windows every quarter.

- Group of institutions

○ Broader range buyer group;

- Cannot commit any consumption volume;

- Funding mechanism with $100 \%$ EC funds;

- All members must be listed;

- Wave 2: vouchers. 
○ Committed buyer group;

- Must commit a consumption volume;

- Single underwriter signs the contract directly with GÉANT;

- Funding mechanism: purchase order, receiving also EC funds (50\% of committed);

- All members must be listed;

- Wave 3: buying services.

Individual institutions and Group of institutions must be listed before the tender comes out in autumn 2019, as OCRE is running and European public procurement system, regulations state that those two types or users must be listed before any tender document gets public. The current deadlines for individual institutions and buyer groups are already closed.

Important to note that, as seen in previous subsection, 39 of the European National Research and Education Network (NREN) registered in the OCRE tender. Hence, all institutions belonging to their NREN are eligible and can request funding for their research activities, despite the fact that the institution itself had not been registered in OCRE by their own. With this the registered NRENs enabled all their members to request funds from OCRE for their research.

\section{2-3- Funding Waves}

There are already as aforementioned 3 waves, which different volume of funding and target users. A preliminary distribution of the total EC funds is shown in Figure 3, which can vary to maximize the utilization of funds. Wave 1 started already in autumn 2019, with first bulk of 500k euro in vouchers for using cloud services (IaaS, SaaS and $\mathrm{PaaS}$ ) with selected providers that already have a contract in place with GÉANT from previous projects.

In 2020 waves 2 and 3 will start consuming services that OCRE will on-board from the companies successfully replying to the two different tenders that will take place, one for cloud and one for Earth Observation services.

Buyers group in wave-3 will benefit from a 50\% co-founding. OCRE will take care, with the help of external experts of the faire distribution of resources.

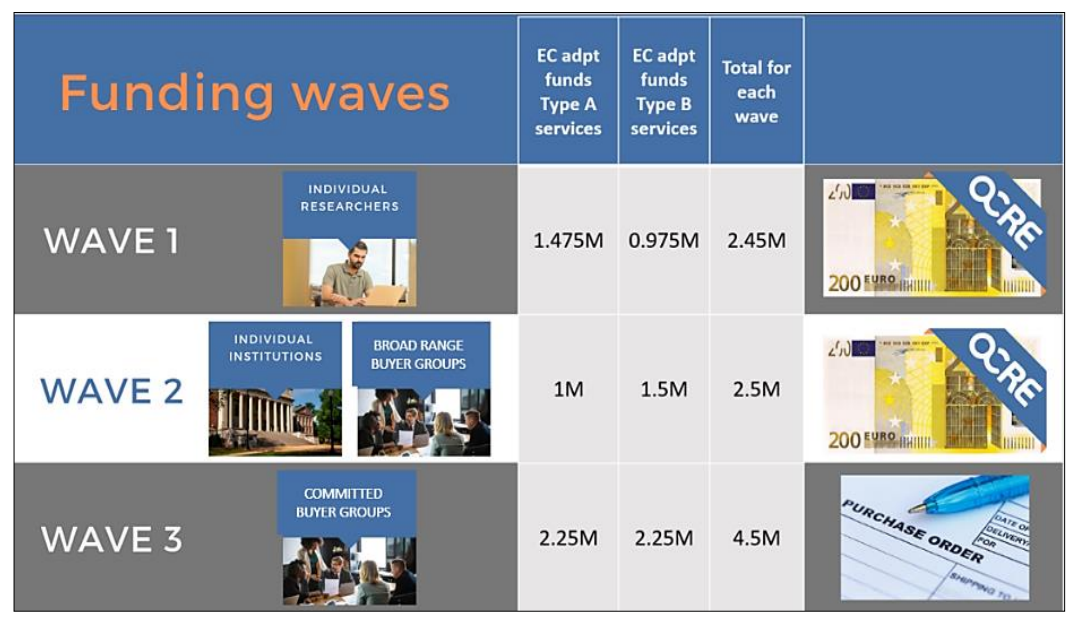

Figure 3. Users, services and preliminary allocation of funding (Meuro).

\section{2-4- Requirements Gathering}

The OCRE team have some of the partners in the consortium with large experience in the Earth Observation domain, from both supplier and researchers side. From one side, the European Association of Remote Sensing Companies (EARSC) which is in direct contact with most of the European companies of the sector, able to engage them and share with them information about our project, gathering also their point of view and concerns. From the other side, RHEA Group has an extensive expertise acquired while working in the Exploitation Platform program for the European Space Agency regarding researchers in the Earth Observation domains, companies and also a very good overview of the last technological solutions employed for providing EO solutions on the digital service offering, such as platforms, datacubes, advanced services and applications, etc.

Starting from the team's robust knowledge of Earth Observation, OCRE launched a requirements gathering campaign after the project start, in order to ensure that the EO services that will be accepted and on-boarded in OCRE would be later used by the research community, ensuring usable and useful services. 


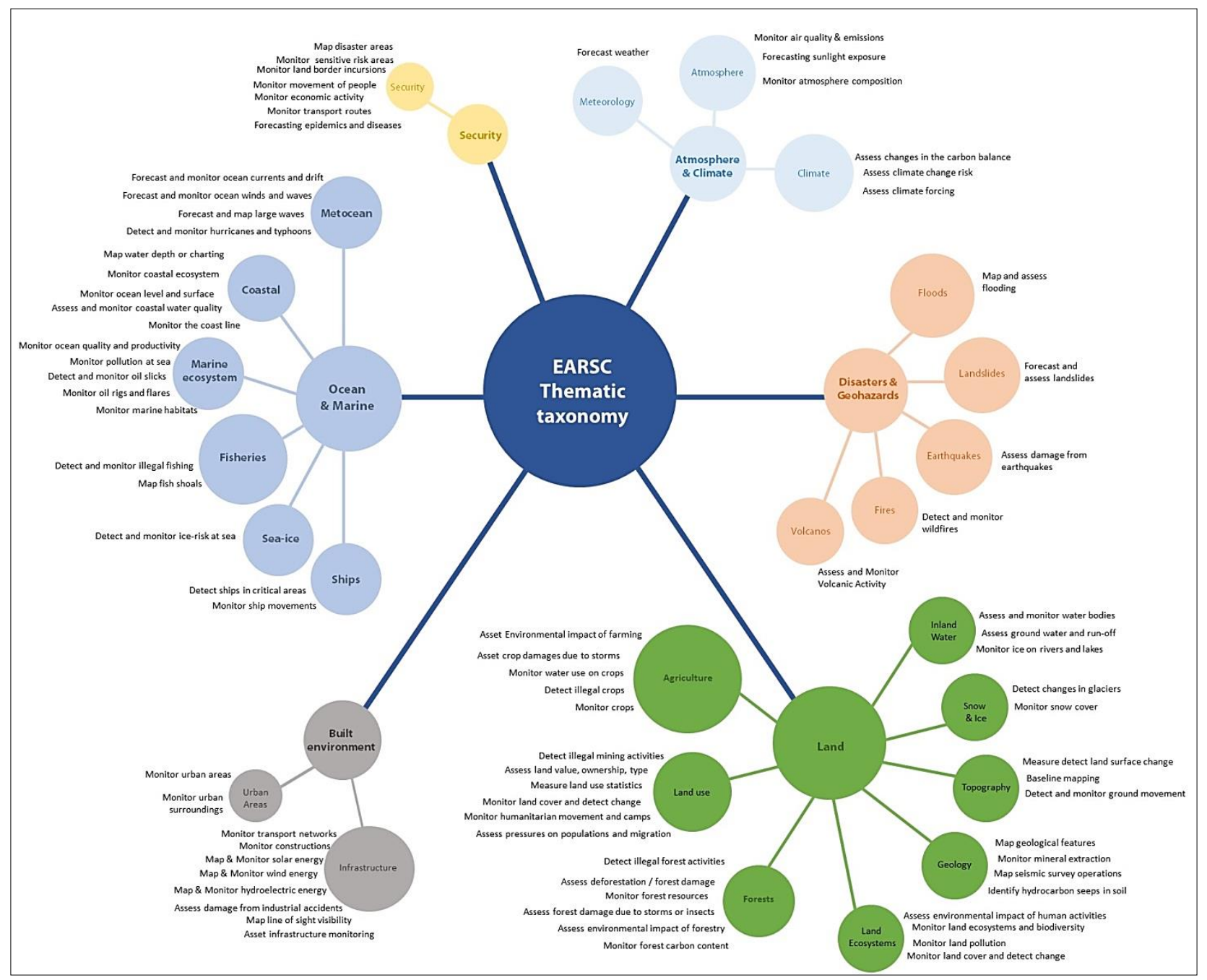

Figure 4. EARSC Thematic taxonomy [1].

\section{2-4-1-Methodology Employed for the Earth Observation Requirements Gathering}

In the frame of the OCRE H2020 project, several method for requirements gathering have been applied such as interviews, online surveys and user workshops, providing a wider perspective of the researchers' needs regarding Earth observation services required, which could be on-boarded in OCRE.

The OCRE website page (https://www.ocre-project.eu) contained and orchestrate the information regarding the whole project and also specific news and pages were created to support the requirements gathering activities.

EARSC created in 2018 a market report [20], where the different activities and services provided by the EU companies were grouped by 6 general themes, containing more detail subgroups, leading finally to a specific activities.

The EO domains were defined as matching the EARSC thematic taxonomy described in [1] and illustrated in Figure 4, including the following first level domain groups:

- Atmosphere and Climate

- Built environment

- Disasters and Geohazards

- Land

- Ocean and marine

- Security

OCRE have employed such thematic taxonomy during requirements gathering activities such as in the online surveys and reporting.

In order to collect feedbacks from researches using EO services or providers of such services OCRE put in place a strategy articulated on different streams of activities. 
First, OCRE launched a series of webinar dedicated to EO domain to have a first contact with the main stakeholders, in parallel a series of relevant events have been identified for having a face2face presentation of OCRE and workshops with potential users and providers. In middle April OCRE launched an online survey in order to reach a broad range of users and providers and at last, to refine the requirements collected up to that moment, a set of champion users in the different domains identified in the taxonomy have been interviewed.

In summary the OCRE project divided the EO requirement gathering process in the following activity streams will be described in more details in the following sections:

- Dedicated Webinars

- Workshops and face to face events

- Online Surveys

- Champion users interviews

\section{2-4-2- Dedicated Webinars}

Since the beginning of the project and in a regular basis, the OCRE project presented the project to researchers and service providers via online webinars, where participants could listen about the news and planning of the project, and during the last part of the webinar could address questions directly to the presenters.

Information about the material and the recorded webinars can be found on the "Events" section of the OCRE website (https://www.ocre-project.eu/events). An example of the webinars announcements is shown in Figure 5.

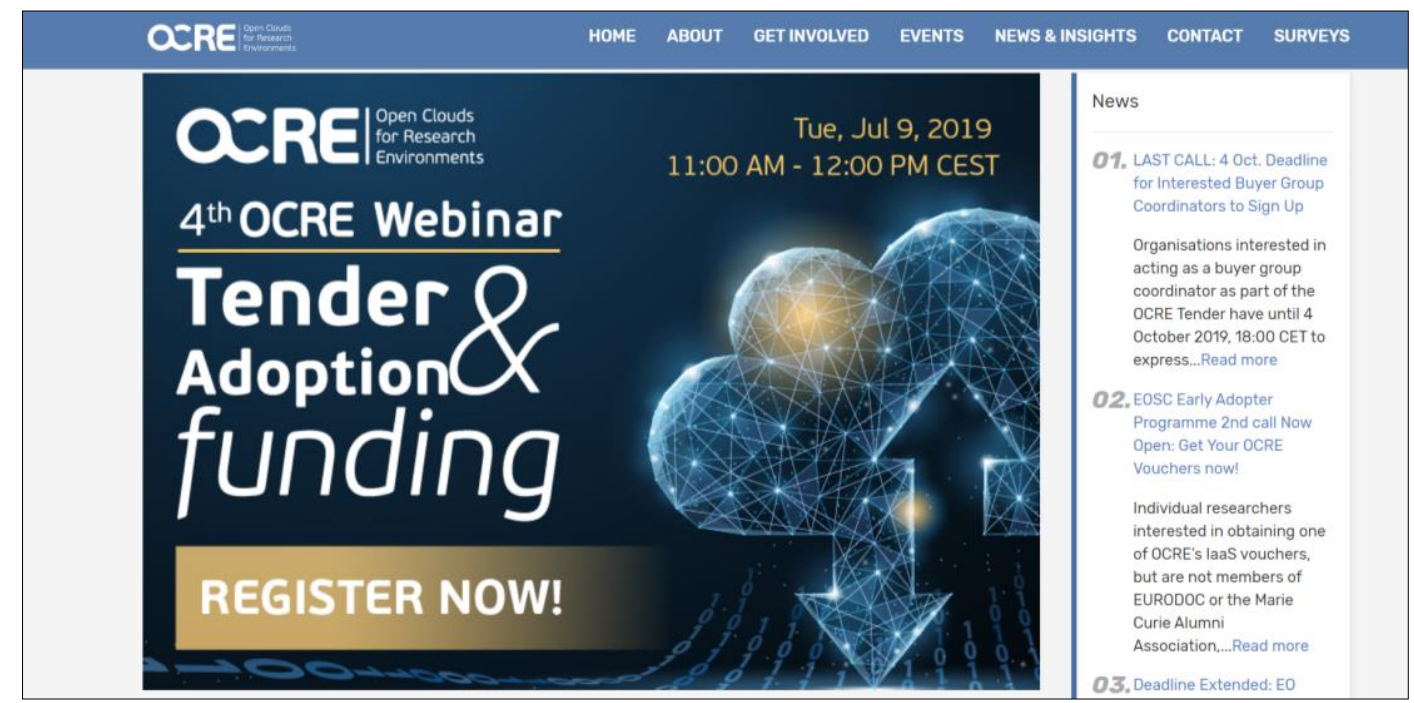

Figure 5. Announcements on the OCRE website regarding the 4th organised Webinar.

\section{2-4-3- Workshops and Face to Face Events}

The OCRE team had participated in a several workshop and conferences dedicated to the Earth Observation domain and not only. Additionally, the team organized dedicated sessions to get direct contact with users and service providers.

These activities aimed to engage users and discuss requirements, promote surveys and eventually stablish direct discussions with the researchers and/or suppliers.

List of EO related events where OCRE has participating since the beginning of the project.

- OCRE F2F meeting, Utrecht, The Netherlands, 12 March.

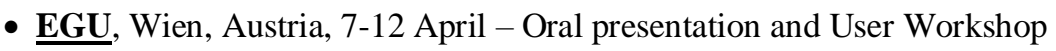

- Living Planet Symposium 19, Milan, Italy, 13-17 May - Agora and poster session, F2F meetings

- EXPANDEO, Brussels, Belgium, 20 June

- EARSeL, Salzburg, Austria, 1-4 July

- Phi Week, Frascati, Italy, 9-13 September

- ISDE 11, Florence, Italy 24-27 September 


\section{2-4-4- Online Surveys}

The online surveys were opened after our intervention at the EGU General Assembly 2019 held in Vienna from $7^{\text {th }}$ to 12th April. However, the questionnaire was already prepared and distributed during our User Gathering workshop done under a Splinter meeting on Friday $12^{\text {th }}$ April, there in EGU19.

The online surveys were available through the OCRE website are they were announced using social media (Twitter, LinkedIn) and during our conference presentations. An example of how the online surveys were announced during conferences is illustrated in Figure 6Figure 6.

The online surveys finished with the raffle of a drone as price on September 13th (last day of Phi-Week 2019) This was needed for motivating the people to participate in the requirements gathering activities. Questionnaire was defined using the RHEA's background knowledge gained while working in the Exploitation Platform program for ESA and the EARSC thematic taxonomy.

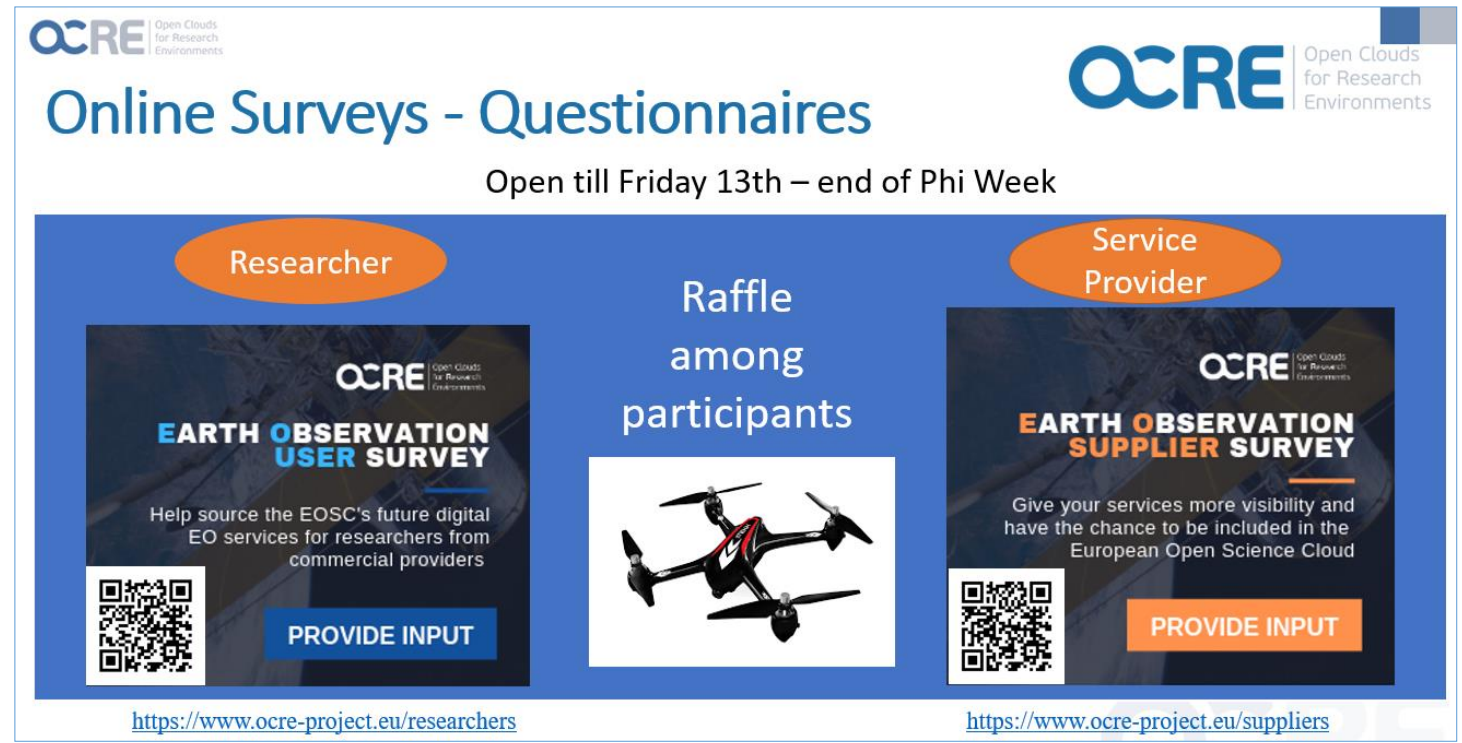

Figure 6. Announcement of the online surveys during conference presentation.

The questions were meant to create a starting point of discussion at the same time that allowing us to understand the main characteristics of the addressed people, as well as their needs and their concerns. They can be answered by EO and non-EO researchers, and they start from a general perspective and follow to understand in higher detail how OCRE and the services that the project will on-board could help the researchers, facilitating them to have more time for developing research and dedicate less effort in basic data processing efforts.

Additionally, and just outreach to raise awareness, the OCRE team launched 2 polls on twitter. The polls are illustrated in Figure 7, the first one (Figure 7, left) in an earlier requirements gathering stage while the second one (Figure 7, right) was when the deadlines for the National Research and Education Network (NRENs) registration was nearby, as the NRENs play a relevant role in the OCRE project.

\begin{tabular}{|c|c|}
\hline OCRE $\begin{array}{l}\text { OCRE } \\
\text { @OCREproject }\end{array}$ & Segui \\
\hline \multicolumn{2}{|c|}{$\begin{array}{l}\text { \#OCRE poll for \#researchers and users of } \\
\text { \#EarthObservation \#Cloud Services: } \\
\text { How much of your \#research is based on \#EO } \\
\text { \#satellite \#data processing or derived } \\
\text { information? } \\
\text { Give us your inputs on the services you would } \\
\text { like to see in the \#EOSC: bit.ly/2QddSa1 \#GIS } \\
\text { \#mapping }\end{array}$} \\
\hline \multicolumn{2}{|l|}{$44 \% 0-25 \%$} \\
\hline \multicolumn{2}{|l|}{$11 \% 25-50 \%$} \\
\hline \multicolumn{2}{|l|}{$11 \% 50-75 \%$} \\
\hline $34 \% 75$ & \\
\hline
\end{tabular}

$$
\begin{aligned}
& \text { ORE OCRE } \\
& \text { @OCREproject } \\
& \text { \#OCRE poll for \#researchers or users of digital } \\
& \text { \#EarthObservation \#EO tools, is your home } \\
& \text { organisation affiliated with any national research and } \\
& \text { education network (\#NREN)? } \\
& \text { \#GIS \#SatelliteData \#EO \#EOdata \#remotesensing } \\
& \text { Yes } \\
& \hline \text { No } \\
& \hline \text { Don't know }
\end{aligned}
$$

Figure 7. Twitters raising the awareness of the OCRE project. 


\section{2-4-5- Champion Users Interviews}

In order to validate and refine the requirements collected with the other activities, OCRE decided to interview a set of champion users relevant in their research domain, according to EARSC taxonomy, and with expertise in the use of Earth Observation data.

The adopted approach for the interview was a Skype call, in which prior to starting with the specific questions, it was shown the OCRE project itself and the benefits that will bring to the EU research environment, from all user point of view, individual researcher, individual institutions and group of institutions so called buyer groups.

Some questions, ranging from the general information about the usage of cloud processing services, amount of research based on EO data or average time spent in standard data processing up to more specific questions about the researchers' trust in commercial services.

The main discussion points were:

- Processing resources employed;

- Common standard processing employed in the data preparation research phase;

- Reliability and trust on commercial offering;

- Quality of the commercial services;

- Research field dependency for using commercial offering;

- Support offered by companies;

- Type of services that will be available;

- EC funds, how to obtain it, for how long, what will happen after OCRE.

\section{3- Requirements Gathering Results}

Regarding the suppliers' participation, the survey got participation from program, account, business and sales managers, including CEO and company president, highlighting the importance of the OCRE project for their business and future activities. A variegated range of companies had participated in our survey from very new EO companies (start-ups) to much more consolidated companies in the EO sector.

For their answers, most of the companies have intermediate of expert experience regarding the usage of HPC, Cloud and Virtualized environment in their activities (see Figure 8).

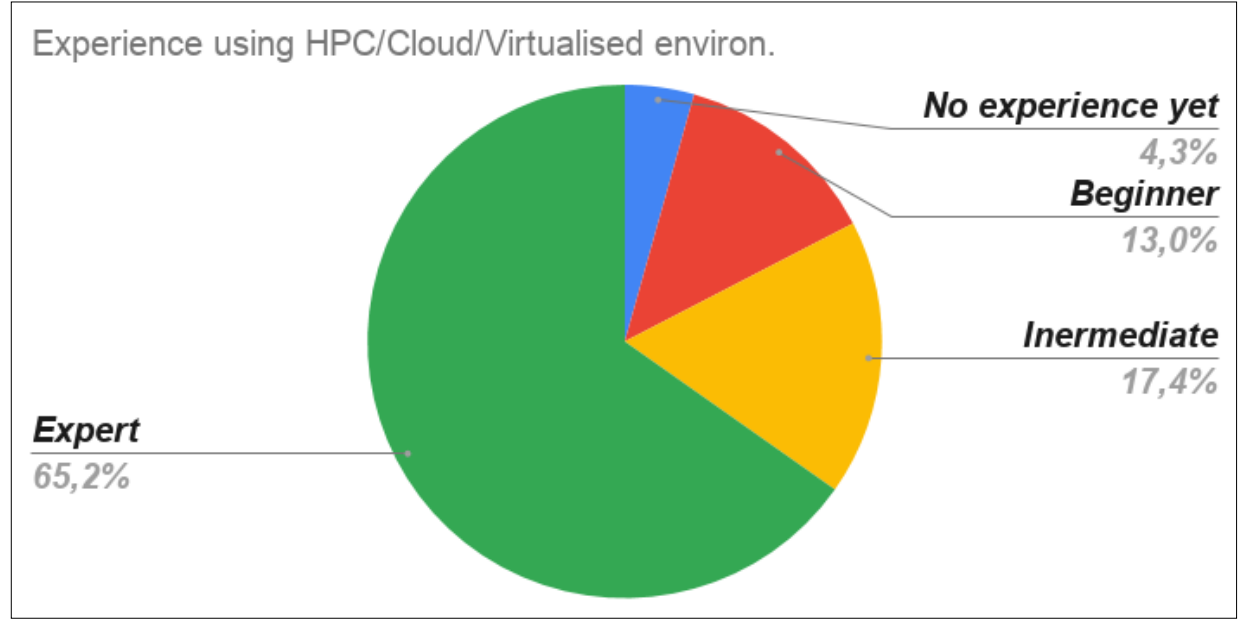

Figure 8. Suppliers' experience using HPC, Cloud and Virtualised environments.

In the suppliers' survey, OCRE wanted also to understand which kind of services are currently offered, and hence, the questionnaire was based on the EARSC classification of EO services based on keywords identifying the main activities, such as assess, detect, forecast, identify, map, measure and monitor. A full list of EO services was reported, reaching a total of 249 services being the applications with more service offering infrastructure, agriculture, forest and land use, as illustrated in Figure 9. 


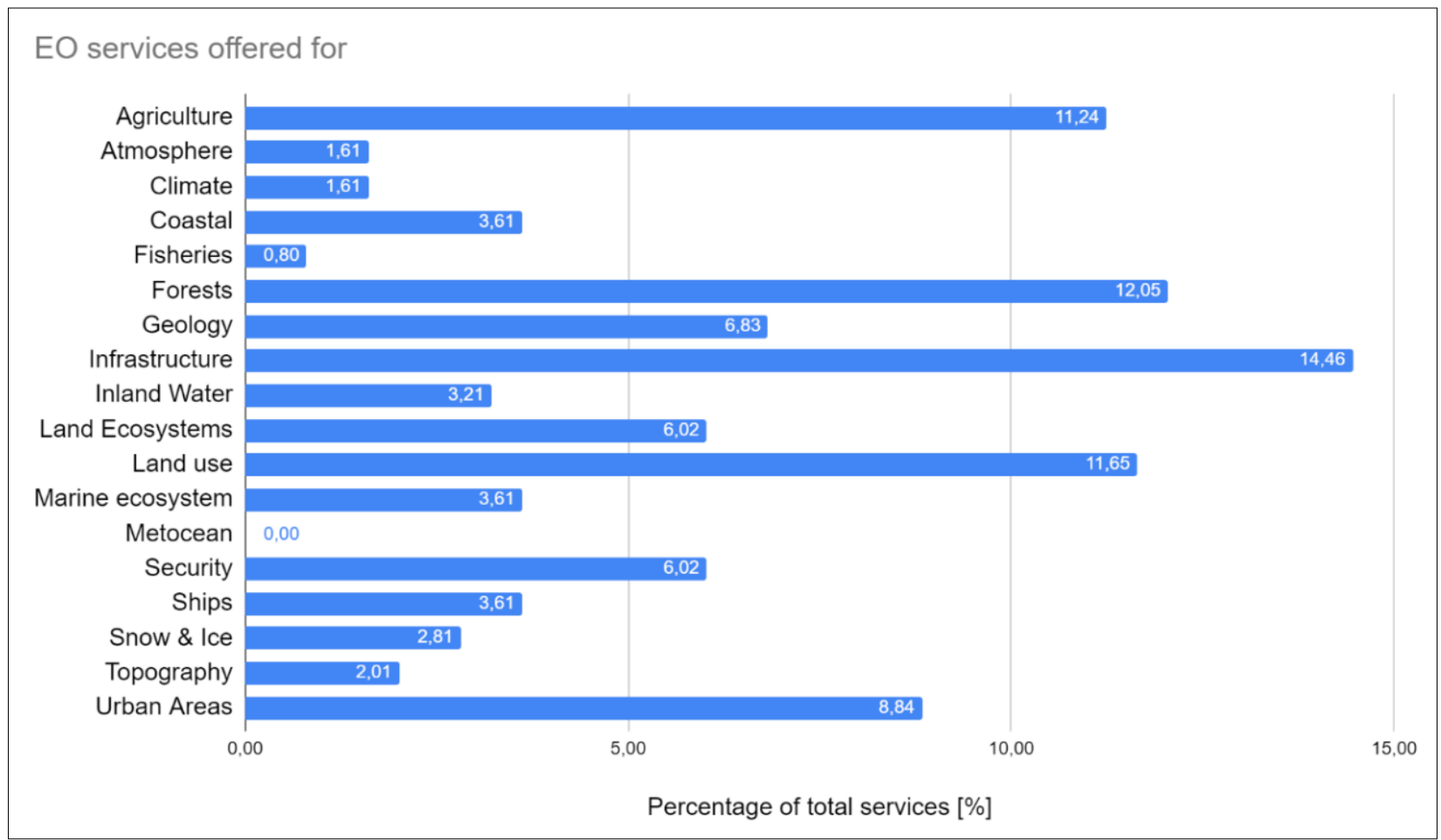

Figure 9. EO services offered under the different subdomains.

\section{3-1-Information Extracted from the Researchers' Surveys}

In our online survey had participated researchers from different position, being dominants the researchers followed by scientist and $\mathrm{PhD}$ students. Over the $76 \%$ of them have experience in using cloud services, with only $9.5 \%$ of them considered experts, and 33\% both of them intermediate and beginners (see Figure 10).

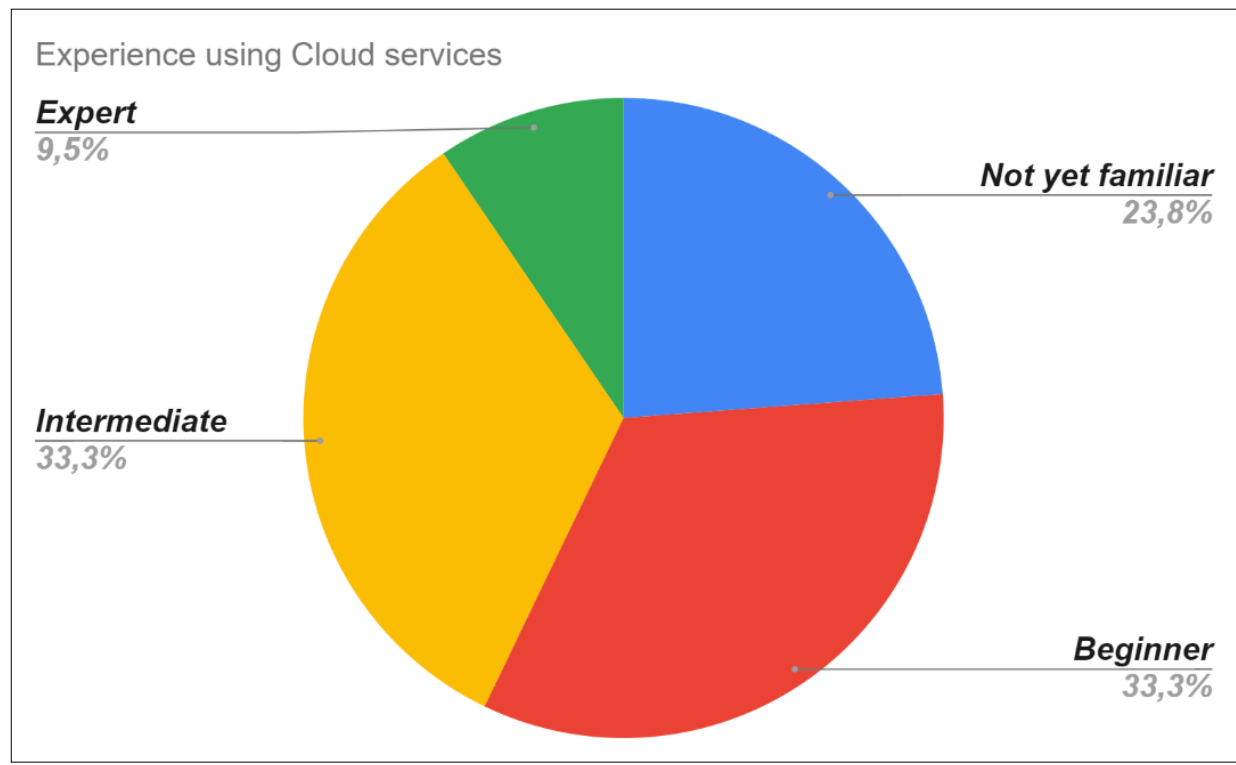

Figure 10. User experience using Cloud services.

OCRE had asked about their familiarity with the Earth Observation data, which results are shown in Figure 11. From the total participation in the researchers' survey, the majority replying are $(57.1 \%)$ considered experts in EO, while the other groups are equal with a $14.3 \%$ each.

These researchers based their research in EO data differently (as shown in Figure 12), where 19\% of the researchers based their research a $100 \%$ in EO data and products, a $42.9 \%$ based their research on a $75 \%$ on $\mathrm{EO}$ data, while $19 \%$ of the total based their research just a little $(25 \%)$ or not in EO products $(0 \%)$. 


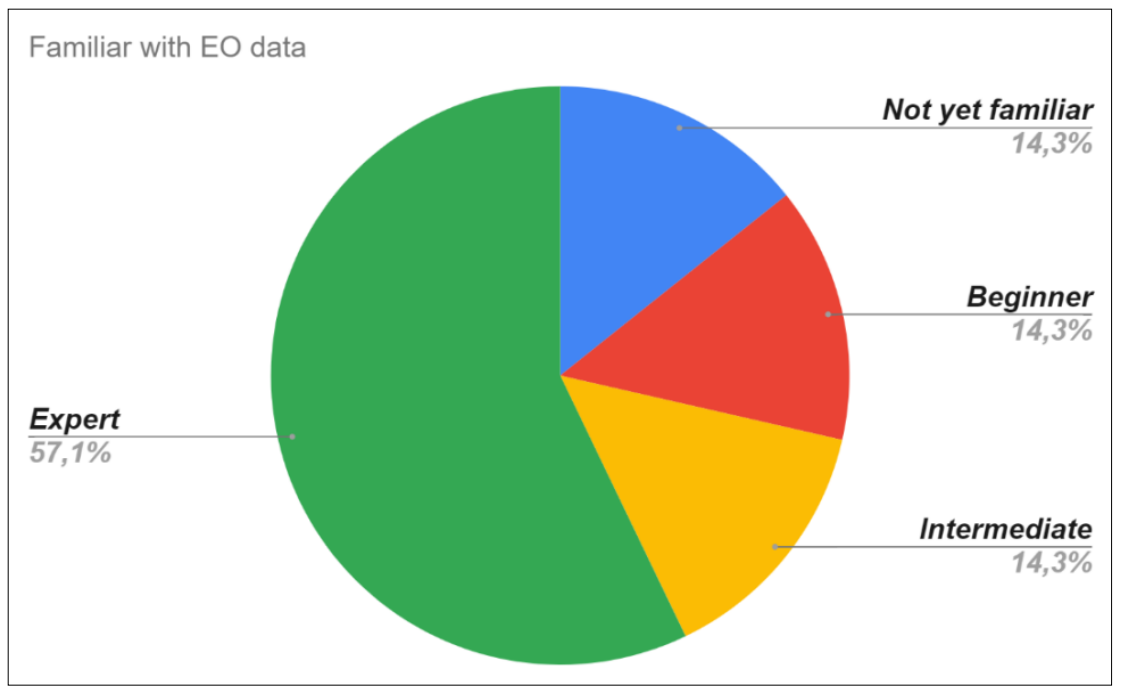

Figure 11. Familiarity of users with EO data.

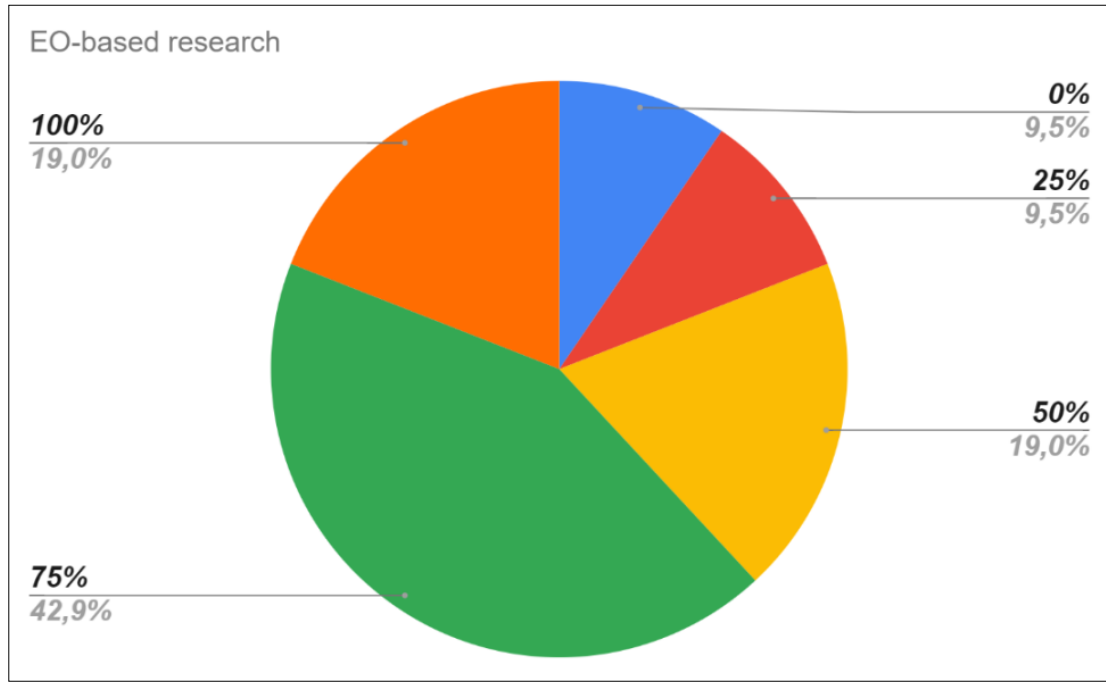

Figure 12. EO-based research of the researchers that had taken the online survey.

We have illustrated common information about EO domain and subdomains represented in the online surveys. All subdomains are covered within the online survey, as illustrated in Figure 13, with a higher representation of the Land and Disasters domains. The main five subdomains are agriculture, urban, forest, land change and floods (Figure 13).

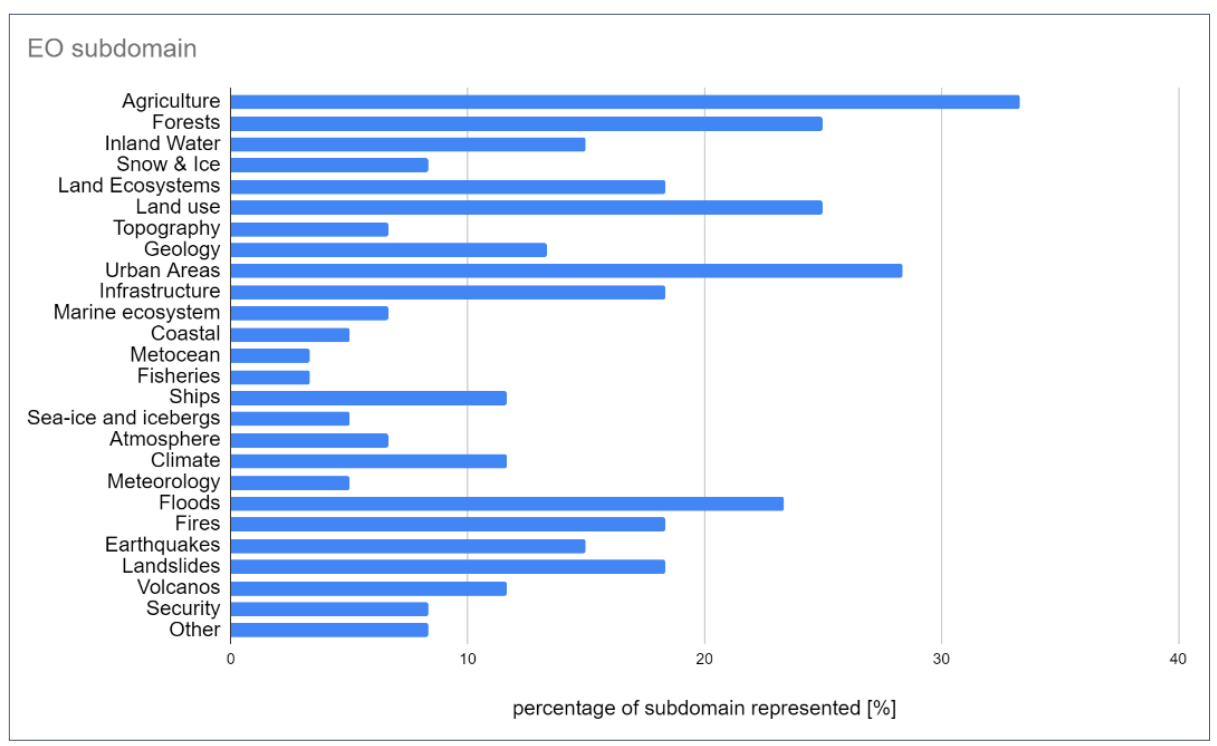

Figure 13. Aggregation of EO subdomain representation (users and suppliers). 


\section{3-2-Champion User Interview Results}

In this subsection, we can find specific information about the outcome of the interviews, regarding research domains, services requested and main EO data employed in their research, illustrated in Figure 14-16, from which the main results are summarized at the end of this subsection.

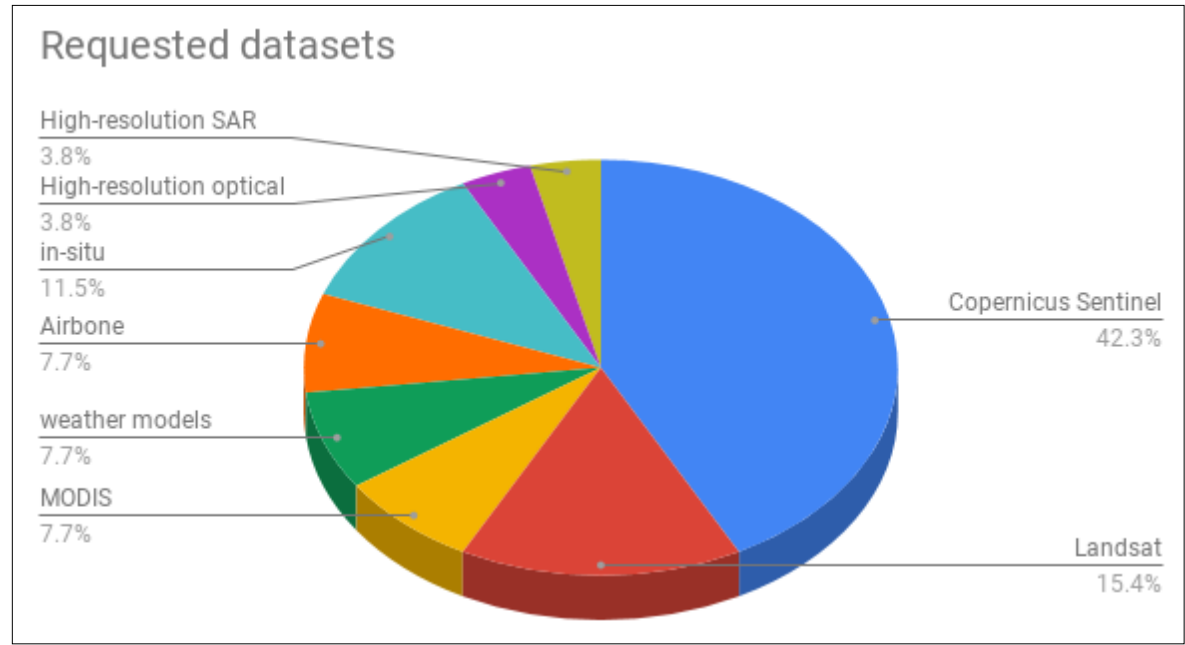

Figure 14. Requested datasets by the interviewed researchers.

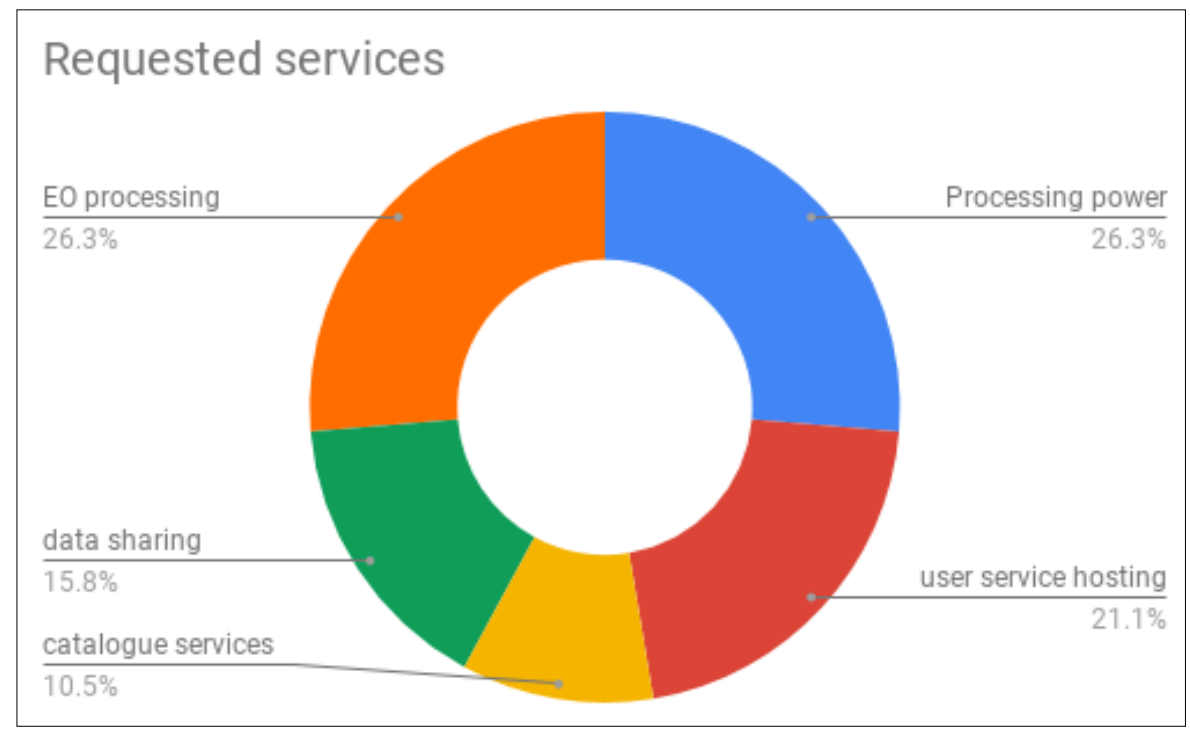

Figure 15. Requested services by the interviewed researchers.

\section{Current processing resources employed}

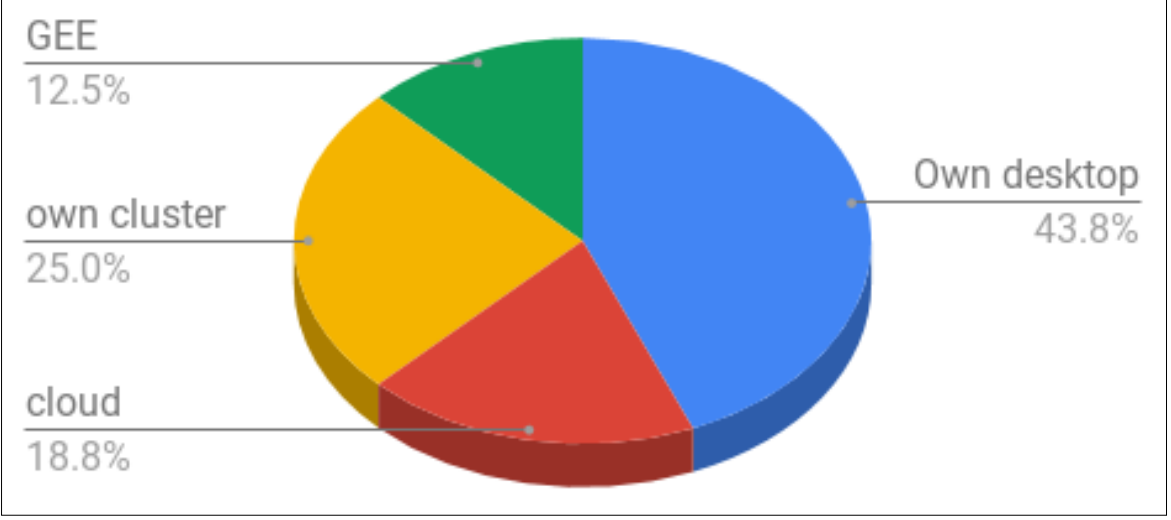

Figure 16. Current processing resources employed by the interviewed users. 
From the results shown in this subsection, the OCRE team can summarize that the Copernicus Sentinel satellites open an era of massive satellite data with almost global daily observations, but for many researchers, other third party data is still needed, such as data from USGS (Landsat, MODIS, etc.), weather models, and high resolution satellite data (see Figure 14). Obviously, the satellite/in-situ data dependency is directly linked to the research domain.

The Figure 15 illustrates the requested services grouped in 5 main categories, which vary depending of the main researcher scope. For example, researchers, which research aim in improving calibration or define new processing algorithms are not suitable for using EO commercial services, which are normally based on recognised standard processing algorithms and workflows. However, these researchers are opened to use commercial services to exploit cloud computing, scalable infrastructure, direct data access to the Copernicus data or even user algorithm hosting or interactive developing services that could facilitate the aforementioned computing capabilities. However, other researchers not focused on the improvement of algorithms or processing workflow, but on large data analytics (i.e. climate change, environmental changes, etc.) are willing to use EO services provided by OCRE, not only processing capabilities related, but Value Added products, or even online data analytics and result sharing.

Finally, as seen in Figure 16 the interviewed researchers still use their own desktop or cluster, being still over the $30 \%$ of researchers experiencing with cloud processing and Google Earth Engine. Normally a factor linked to their age and their ability to adapt and use new technologies.

From the different interviews, we can summarize researchers' needs coming from a wide range of research fields and domains and group them in specific identified use cases covering most of the researchers' needs.

\section{3-3- Total Participation Overview}

The total participation counts with 19 EU countries replying and participating to our requirements gathering activities covering all 6 thematic groups and all their subdomains, with 25 subdomains in total.

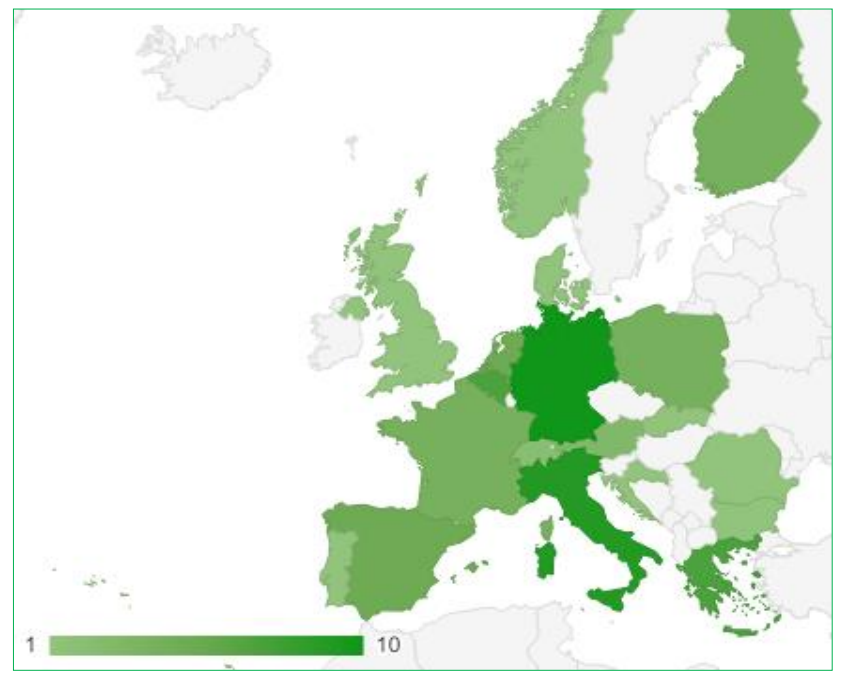

Figure 17. Geographic distribution of the total participants in the OCRE requirements gathering.

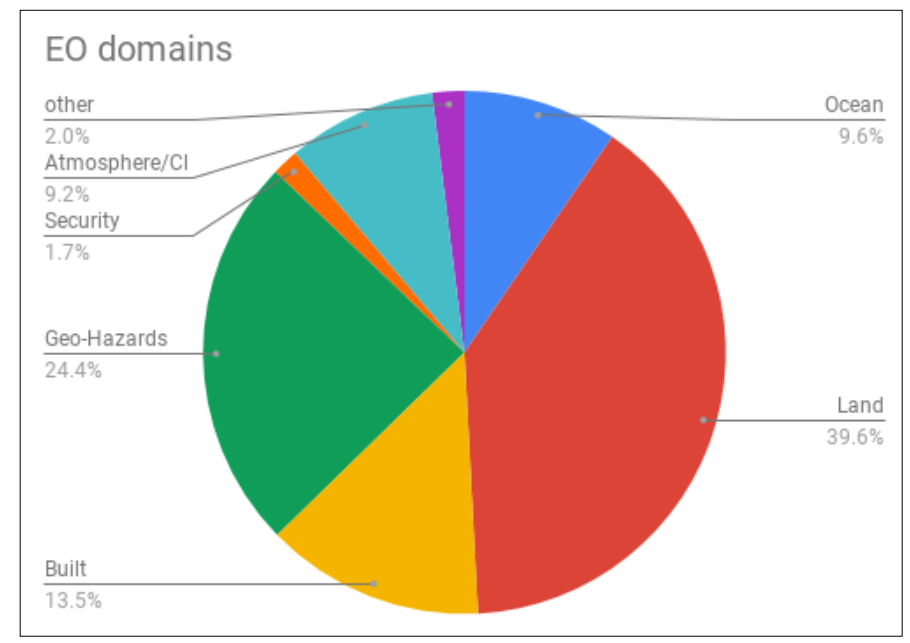

Figure 18. EO domains represented by the total participation in the requirements gathering. 


\section{3-4-Ranking of Requirements and Identified Use Cases}

From the requirements gathering activities, OCRE identified a ranking or requirements coming from researchers involved in Face2Face meetings, workshops, conferences, online surveys and interviews.

This ranking of requirements is:

1. Processing with collocated data (the game changer role of the DIASes)

2. EO data processing

3. User algorithm hosting

4. Interactive algorithm development

5. Data available/provided by the EO suppliers

6. Data analytics

7. Data sharing capabilities

8. On-demand and online services easy to use

9. Support and training.

The requirements gathering can conclude that there are different specific applications for different themes but all have in common the following general use cases:

- EO data processing

- User algorithm hosting

- Interactive algorithm development

- Value added products

- Data analytics

- Data sharing capabilities

The market offer various solutions with support to the different grouping of the use cases above so a service covering even one of the scenario above would be of benefit for users.

\section{3-5-Framework Agreements}

In Autumn/Winter 2019 both tenders, one for cloud services and one for Earth Observation services should come out, and during the Q1/Q2 of 2020 the granted services should be ready to use. Contracts will be signed directly with GÉANT.

Prior the tenders are published, OCRE provides publicly in its website the Prior Information Notice documents, so the companies can have the chance to check the preliminary requirements and will have time to provide a feedback on their points. Both PINs and tender documents will come separately for cloud and EO services.

\section{3-6-Cloud and EO Service Consumption}

Service consumption will be monitored through the technical management platform which purpose is the activation, monitoring and reporting of the distributed founding, some of which will be in the form of vouchers for individual researchers.

Vouchers will be requested by individual researchers and screened by known scientific organisations such as Marie-Curie Alumni Association (MCAA) and Eurodoc as well as the EOSC-hub early adopter program. The first two organisations will screen and distribute vouchers among their own members that will request them, and the last one, open for the rest of individual researchers. Additionally for 2020 will be another institution screening and distributing vouchers specifically for the Earth Observation community.

Vouchers will be only consumed for the specific service which was requested, and cannot be exchange for money or any other way of funding. All distributed vouchers will have an indicated expiring date, which will not be longer than the duration of the OCRE project, which will end on $31^{\text {st }}$ December 2021.

\section{4- Conclusion}

The European Commission through the European Open Science Cloud initiative, it is funding OCRE project to stimulate the usage of commercial services by the European research community. The scope of our project is to stimulate the usage of the services that will be granted after the tender evaluation, from where the research community 
will receive funding to use the services for free or with a co-funding from the EC, until the entire usage of the funds or the end of the project (December 2021).

The objective of the OCRE project until now has been to raise the awareness of the project from both researchers and service providers point of view, as it is a funding opportunity for researchers and a good business opportunity for the European service providers of both and Earth Observation services.

OCRE is a pioneer project when for the first time during the Copernicus era, the European Commission provided funds for stimulating the European Earth Observation market, bringing services and researchers towards the Data Infrastructure Access Services, where all the Copernicus Sentinel data is stored, being in operations since autumn 2018.

Analogously, the European Space Agency (ESA) is financing similar project called Network of Resources (NoR) [21] which is exclusive for Earth Observation researchers and commercial applications during the prototype developing phase.

Hence, OCRE and NoR are very ambitious and unprecedented projects, with high impact in the future EO market activities and offers, with the objective to burst the usage of EO commercial services by the research environment, by incorporating such services in their daily activities.

\section{5- Funding}

OCRE is part of the European Open Science Cloud and receives funding from the EC under grant agreement no. 824079 .

\section{6- Conflict of Interest}

The author declares that there is no conflict of interests regarding the publication of this manuscript. In addition, the ethical issues, including plagiarism, informed consent, misconduct, data fabrication and/or falsification, double publication and/or submission, and redundancies have been completely observed by the authors.

\section{7- References}

[1] Popescu, I. What is the European Open Science Cloud? Available online: https://www.egi.eu/about/newsletters/what-is-theeuropean-open-science-cloud/ (accessed on 31 January 2019).

[2] Wilkinson, Mark D., Michel Dumontier, Ijsbrand Jan Aalbersberg, Gabrielle Appleton, Myles Axton, Arie Baak, Niklas Blomberg, et al. "The FAIR Guiding Principles for Scientific Data Management and Stewardship." Scientific Data 3, no. 1 (March 15, 2016). doi:10.1038/sdata.2016.18.

[3] European Commision. EU ministers endorse Commission's plans for research cloud. Available online: https://ec.europa.eu/info/news/eu-ministers-endorse-commissions-plans-research-cloud-2018-may-29_en (accessed on 3 February 2019).

[4] EOSC-Hub. The European Open Science Cloud is officially launched. Available online: https://www.eoschub.eu/news/european-open-science-cloud-officially-launched (accessed on 2 March 2019).

[5] EOSC-Hub. EOSC-hub. Available online: https://www.eosc-hub.eu/ (accessed on 16 February 2019).

[6] Fernández-del-Castillo, Enol, Diego Scardaci, and Álvaro López García. "The EGI Federated Cloud e-Infrastructure.” Procedia Computer Science 68 (2015): 196-205. doi:10.1016/j.procs.2015.09.235.

[7] Lecarpentier, Damien, Peter Wittenburg, Willem Elbers, Alberto Michelini, Riam Kanso, Peter Coveney, and Rob Baxter. "EUDAT: A New Cross-Disciplinary Data Infrastructure for Science." International Journal of Digital Curation 8, no. 1 (June 14, 2013): 279-287. doi:10.2218/ijdc.v8i1.260.

[8] Salomoni, Davide, I. Campos, Luciano Gaido, Giacinto Donvito, M. Antonacci, P. Fuhrman, J. Marco et al. "INDIGODatacloud: foundations and architectural description of a Platform as a Service oriented to scientific computing." arXiv preprint arXiv:1603.09536 (2016).

[9] OCRE. The H2020 OCRE project: Open Cloud for Research Environments. Available online: www.ocre-project.eu (accessed on 1 January 2019).

[10] eInfraCentral. The eInfra Central project. Available online: https://www.einfracentral.eu/home (accessed on 5 April 2019).

[11] EOSCpilot. The European Open Science Cloud for Research Pilot Project. Available online: https://eoscpilot.eu/ (accessed on 15 March 2018).

[12] GÉANT. GÉANT Project (GN4-2). Accelerating research, driving innovation and enriching education. Available online: https://www.geant.org/Projects/GEANT_Project_GN4 (accessed on 7 April 2019). 
[13] OpenAIRE. OpenAIRE - Advance Project. Available online: https://www.openaire.eu/advance/ (accessed on 7 April 2019).

[14] EOSC. EOSC-SYNERGY. Extending EOSC coordination at the National Level. Available online: https://www.eoscsynergy.eu/ (accessed on 7 April 2019).

[15] DEEP-Hybrid Data Cloud. DEEP-Hybrid Data Cloud. Supporting AI post-processing analysis of very large data, and analysis of massive online data streams over distributed e-Infrastructures in the EOSC. Available online: https://deep-hybriddatacloud.eu/ (accessed on 5 May 2019).

[16] European Commission. Co-designed Citizen Observatories Services for the EOS-Cloud: The COS4Cloud project. Available online: https://cordis.europa.eu/project/id/863463/es (accessed on 1 December 2019).

[17] EOSC-Hub. EPOS-ORFEUS Competence Center. Available online: https://www.eosc-hub.eu/research-communities/eposorfeus-competence-center (accessed on 1 December 2019).

[18] EOSC-Hub. The Dynamic On Demand Analysis Service (DODAS). Available online: https://www.eosc-hub.eu/services/ Dynamic on Demand Analysis Service (accessed on 1 December 2019).

[19] EOSC-Hub. The European Network for Earth System Modelling (ENES). Available online: https://www.eosc-hub.eu/eoscpractice-enes (accessed on 1 December 2019).

[20] The European Association of Remote Sensing Companies (EARSC). A Taxonomy for the EO Services Market: enhancing the perception and performance of the EO service industry. Available online: http://earsc.org/file_download/489/ A+Taxonomy+for+the+EO+Services+Market+issue+2+\%282\%29.pdf (accessed on 2 February 2019).

[21] European Space Agency. The EO Network of Resources (NoR) ESA project. Available online: https://eo4society.esa.int/projects/eo-network-of-resources/ (accessed on 2 December 2019). 